Wohnungspolitik: »[S]ie werden nicht gehört. [...] [W]enn Ihnen da irgendjemand [...] in irgendeinem Ministerium etwas anderes sagt, mit Verlaub, dann sagt er Ihnen nicht die Wahrheit « (IP 52: 59ff.).

Diesbezüglich zeigen die Problemstrukturen, dass die Auswirkungen des demografischen Wandels auf die Wohnungsmärkte in den 2000er und frühen 2010er Jahren durchaus aktiv politisch adressiert worden sind (vgl. Kapitel 7.2.2). Seit etwa Mitte der 2010er Jahre dominieren hingegen die Wohnraumknappheit und Bezahlbarkeit die Wohnungspolitik. Für den barrierefreien bzw. -reduzierten Wohnraum stellt diese Entwicklung ein Unmöglichkeitsfenster dar, was das Agenda-Setting und die Politikformulierung im Sinne einer stärkeren politischen Verankerung erschwert:

»Also, ich glaube im Moment ist [...] der Zug so ein bisschen abgefahren. Also, man hätte es vielleicht seinerzeit, als das aufkam, stärker in Angriff nehmen müssen, dann wäre es jetzt präsent. Aber man hat es nur so niederschwellig gehalten und deswegen sind jetzt andere Themen wie das Energetische oder überhaupt, dass Wohnungen fehlen, auf einmal viel wichtiger, als eben diese Barrierearmut« (IP 13: 61).

\title{
7.3 Vergleichende Betrachtung und theoretische Rückbindung
}

Bislang wurden die politischen Prozesse zum barrierefreien bzw. -reduzierten Wohnraum in Sachsen-Anhalt und Hessen getrennt analysiert. Forschungsleitend war die Frage B des Erkenntnisinteresses: Was erklärt das Zustandekommen bzw. Ausbleiben sowie die konkrete Ausgestaltung dieser Policies? Abschließend sollen die zentralen Erkenntnisse aus den beiden Fallstudien zusammengefasst und verglichen werden, wiederum orientiert an den fünf AEP-Erklärungsfaktoren. Wo finden sich landesspezifische Differenzen, wo bestehen Indizien für übergeordnete Muster?

\subsubsection{Instrumente und Instrumenten-Alternativen}

Der erste Erklärungsfaktor wurde in Policies im bestehenden Policy-Mix einerseits und Instrumenten-Alternativen andererseits differenziert (vgl. Kapitel 4.2). Im Anschluss an eine diesbezügliche Zusammenfassung sind die sogenannten ideologischen und institutionellen Filter zu diskutieren, die politische Maßnahmen auf dem Weg zu ihrer Durchsetzung zu durchlaufen haben.

Policies im bestehenden Policy-Mix

Der untersuchte Policy-Mix zeigt landesspezifische Besonderheiten. So wählt SachsenAnhalt im Bauordnungsrecht eine enge Anlehnung an die Musterbauordnung, während Hessen mit der 20-Prozent-Regelung einen Sonderweg formuliert und zugleich orientiert an dem Bundesmuster des Jahres 2016 die zuvor bestehende rollstuhlgerechte Zugänglichkeit streicht. Trotz hessischem Sonderweg ist das ARGEBAU-Muster für die politischen Prozesse in beiden Bundesländern ein zentraler Orientierungspunkt, Abweichungen davon bedürfen Begründungen. Damit werden die Änderungen in der 
Hessischen Bauordnung von Behinderten- und Sozialverbänden auch deutlich als Rückschritt in Sachen barrierefreies Bauen kritisiert (vgl. Kapitel 7.3.5)

In der Wohnraumförderung unterstützt Sachsen-Anhalt den barrierereduzierenden Umbau von Bestandsgebäuden insbesondere durch das AUFZUGS-Programm, aber auch durch Sachsen-Anhalt MODERN und WOHNRAUM HERRICHTEN. Die hessische Wohnraumförderung ist breiter aufgestellt und denkt je im Neubau und im Bestand sowie je für selbstnutzende Eigentümer*innen und für den Mietwohnraum Barrierefreiheit bzw. -reduzierung mit. Gleichzeitig sind diese Programmbestandteile weniger prominent, lediglich das Zuschussprogramm zum behindertengerechten Umbau gilt als einschlägige Förderung. Dieses Programm wurde aufgestockt und in der Mietwohnraumförderung eine Aufzugsförderung integriert, gleichzeitig entfiel die Förderung für barrierefreien und eingeschränkt mit dem Rollstuhl nutzbaren Wohnraum. Zudem wirken die Erfahrung mit früheren Förderprogrammen nach: So gab es in Sachsen-Anhalt in den späten 1990er Jahren eine Förderung von barrierefreien Eigenheimen und in Hessen Zuschüsse für den behindertengerechten Umbau von Mietwohnungen. Beides wurde von den damaligen Wohnungsmärkten nicht stark nachgefragt, weshalb die Wiedereinführung vergleichbarer Förderprogramme heute schwierig ist (vgl. institutioneller Filter, Kapitel 4.1).

Für die Wohnberatung zur Wohnungsanpassung besteht in Sachsen-Anhalt mit PiA e.V. ein Verein, der vom Sozialministerium gefördert wird. In Hessen gibt es eine einschlägige Fachstelle für die Ausbildung, Weiterbildung und Vernetzung im Land. Die Wohnberatungen selbst sind in beiden Bundesländern vor allem über kommunale Beratungen oder freie Träger organisiert. Ergänzt wird die informationelle Steuerung über eine Fach-/Kompetenzstelle für Barrierefreiheit. Eine solche Stelle wurde in SachsenAnhalt im Frühjahr 2020 etabliert und bestand zuvor als ehrenamtliche Struktur. In Hessen ist sie im Koalitionsvertrag des Jahres 2019 festgeschrieben. In beiden Bundesländern geht die Initiative dazu auf die Aktionspläne zur Umsetzung der UN-Behindertenrechtskonvention zurück, es handelt sich um dezidiertes Disability Mainstreaming in unterschiedliche Ressorts hinein. Diese Fachstellen sind induktiv aus dem empirischen Material abgeleitet worden und es bedürfte einer weiteren wissenschaftlichen Begleitung, um ihre letztliche Policy-Wirkung zu überprüfen.

Instrumenten-Alternativen

Ferner war von Interesse, inwieweit Instrumenten-Alternativen die politische Steuerung beeinflussen. Darunter fallen einerseits die staatliche Bereitstellung von Wohnraum, andererseits kooperative Instrumente über Bündnisse und die wirtschaftliche Selbstregelung im Schatten der Hierarchie (vgl. Kapitel 4.2.1). Zudem können sonstige bundespolitische oder kommunale Maßnahmen den landespolitischen Mix zum barrierefreien bzw. -reduzierten Wohnraum flankieren. Dabei wurde unterteilt in Instrumenten-Alternativen nach Typ 1, die Veränderungen in den bestehenden Maßnahmen beschreiben und unter dem AEP-Erklärungsfaktor »Akteure und ihre Handlungen« analysiert worden sind, sowie Typ 2 als ergänzende Maßnahmen und Typ 3 als den Policy-Mix ersetzende Maßnahmen (vgl. Kapitel 4.2.2). 
Die staatliche Bereitstellung von Wohnraum über kommunale oder landeseigene Wohnungsunternehmen wird weder in Sachsen-Anhalt noch in Hessen aktiv zur Bereitstellung von barrierefreiem bzw. -reduziertem Wohnraum genutzt. Zwar seien politische Zielvorgaben laut den Interviewpartner*innen hier leichter zu integrieren als bei der privatwirtschaftlichen Wohnungs- und Bauwirtschaft, allerdings müssten sich letztlich alle Wohnungsunternehmen der wohnungswirtschaftlichen Logik anpassen. Dies ist eine interessante Erkenntnis, da vielfach auf die wohnungs- und sozialpolitische Relevanz staatlicher Wohnungsunternehmen rekurriert wird (bspw. Schönig et al. 2017b: 40ff.; Landsberg 2017: 4; BBSR 2017: 16ff.). Gleichzeitig erkennen bereits mehrere Studien den Marktzwang auch von kommunalen Wohnungsunternehmen an, verweisen auf ihre gewinnorientierte Gesellschaftsform als $\mathrm{GmbH}$ oder AG und benennen gestiegene Herstellungskosten, die den Bau von kostengünstigen Wohnungen ohne ausreichende politische Förderung erschweren bis verhindern (von Einem 2016a: 30f.; von Einem 2016b: 168; Schönig et al. 2017b: 40).

Eine weitere Instrumenten-Alternative besteht in kooperativen Instrumenten über Bündnisformate sowie die wirtschaftliche Selbstregelung. Die lokalen Netzwerke in SachsenAnhalt diskutieren dabei eher kommunale Vorhaben und die hessische Allianz für Wohnen fokussiert klar den bezahlbaren Wohnraum. Relevanter für den barrierefreien bzw. -reduzierten Wohnraum sind Formen der wirtschaftlichen Selbstregelung, wobei die Wohnungs- und Bauwirtschaft im sogenannten hierarchischen Schatten auf veränderte Wohnraumbedarfe reagiert. Diesbezüglich besteht in Sachsen-Anhalt durch den demografischen Druck auf den Wohnungsmärkten ein größeres Interesse zum Handeln, wohingegen die Wohnungs- und Bauwirtschaft im hessischen Rhein-Main-Gebiet derartigen Zusatzbedarfen verhaltener begegnet. In beiden Bundesländern argumentiert die Wohnungs- und Bauwirtschaft allerdings mit solchen eigenen Aktivitäten gegen strengere regulative Vorgaben (vgl. Instrumenten-Alternative, Typ 3), wohingegen ökonomische Anreize sowie informationelle Angebote die wirtschaftliche Selbstregelungsfähigkeit unterstützend ergänzen (Instrumenten-Alternative, Typ 2). Dabei besteht sowohl in Sachsen-Anhalt als auch in Hessen eine Schwerpunktsetzung auf die wachsende Kundengruppe der älteren Menschen und das mittlere bis höhere Preissegment. Entsprechend ist ein Marktversagen, das verbindliche politische Maßnahmen begründen könnte, in zweierlei Richtungen möglich: einerseits bei Personengruppen mit speziellen Wohnraumbedarfen und andererseits bei der Herstellung von barrierefreiem bzw. -reduziertem und zugleich bezahlbarem Wohnraum, vor allem im Rahmen sozialstaatlicher Transferleistungen.

Weitere Instrumenten-Alternativen auf Bundesebene oder auf Ebene der Kommunen können den landespolitischen Policy-Mix flankierend erweitern (InstrumentenAlternative, Typ 2). Während in Sachsen-Anhalt vereinzelte Bundesaktivitäten wie Modellprojekte oder mietrechtliche Regelungen genannt werden, bescheinigen Interviewpartner*innen in beiden Ländern der kommunalen Ebene weitreichendere Handlungsspielräume. Über städtebauliche Verträge, Förderprogramme sowie Seniorenund Pflegestützpunkte seien wohnungspolitische Zusatzbedarfe zielsicher lokal zu steuern. In Hessen kommen solche Maßnahmen bereits stärker zur Anwendung, wohingegen für Sachsen-Anhalt eher von potentiellen Instrumenten-Alternativen gesprochen wird. 
Ideologische und institutionelle Filter in der Instrumentenwahl

Instrumente und Instrumenten-Alternativen sind dabei keinesfalls werturteilsfrei, sondern unterliegen akteursspezifischen Policy-Präferenzen, welche die Einführung einer Maßnahme erleichtern oder behindern können (vgl. Kapitel 4.2.1). Deshalb wurden die Interviewpartner*innen gefragt, welche Maßnahmen sie zur politischen Steuerung von barrierefreiem bzw. -reduziertem Wohnraum bevorzugen. Für das unmittelbar bindende Bauordnungsrecht votieren Behinderten- und Sozialverbände, die sich für eine ausnahmslose und strikte Einführung der Barrierefreiheit aussprechen (IP 23: 76; IP 33: 64). Finanzielle Anreizstrukturen seien unzureichend, »dann erreicht man ja nur die, die a) es auf dem Schirm haben, die dann auch noch [b)] Lust haben, sich mehr Mühe zu machen (IP 26: 16). Demgegenüber bevorzugen die Wohnungs- und Bauwirtschaft sowie parteipolitisch CDU und FDP eben eine solche finanzielle Förderung (IP 12: 10; IP 27: 41ff.; IP 28: 19; IP 30: 19; IP 38: 70; IP 41: 6; IP 47: 34; IP 50: 6; IP 53: 46ff). Interviewpartner*innen aus dem politisch-administrativen System begründen ihre Präferenz für weichere Steuerungsformen zudem mit der leichteren politischen Durchsetzbarkeit: "Und das geht seitens des Staates, da Einfluss zu nehmen [...], geht nur über Geld. Weil, eine andere Handhabe - außer, du machst Gesetze und schreibst es genau vor-, da gehen dir alle aufdie Barrikaden « (IP 44: 52, vgl. auch IP 24: 13; IP 45: 8). Folgerichtig werden Beratungsstellen zur Wohnungsanpassung als niedrigschwellige und unverbindliche Maßnahmen einhellig von allen Interviewpartner*innen befürwortet (IP 27: 31ff.; IP 29: 111; IP 30: 31; IP 40: 16).

Die hier skizzierten Policy-Präferenzen wirken als ideologischer Filter, den eine potentielle Maßnahme bis zu ihrer Verabschiedung zu durchlaufen hat. Darüber hinaus bestehen beim barrierefreien bzw. -reduzierten Wohnraum verschiedene institutionelle Filter (vgl. Böcher/Töller 2012: 195; Böcher/Töller 2007: 313f.). So wirken sowohl in Sachsen-Anhalt als auch in Hessen negative Erfahrungen mit früheren Förderprogrammen als Pfadabhängigkeit nach, was etwa eine neuerliche Förderung für barrierefreie Eigenheime erschwert. Darüber hinaus wird die politische Steuerung der Wohnberatungen zur Wohnungsanpassung dadurch behindert, dass für diese Beratungsleistung bislang keine dezidierte Zuständigkeit im föderalen System festgeschrieben ist.

\section{Zentrale Erkenntnisse zu »Instrumente und Instrumenten-Alternativen«}

Der Policy-Mix in Sachsen-Anhalt orientiert sich stärker an übergeordneten Mustern wie der ARGEBAU-Musterbauordnung und dem KfW-Programm »Altersgerecht Umbauen«, wohingegen Hessen eher eigene Policy-Akzente setzt.

Als Instrumenten-Alternative gilt in Sachsen-Anhalt vor allem die wirtschaftliche Selbstregelung, in Hessen eher kommunale Maßnahmen (beides: ergänzende InstrumentenAlternative Typ 2). Teils begründet die Wohnungs- und Bauwirtschaft die wirtschaftliche Selbstregelung als Ersatz für regulative Vorgaben (Instrumenten-Alternative Typ 3).

Instrumenten-Alternativen haben vor ihrer Einführung ideologische und institutionelle Filter zu durchlaufen. 


\subsubsection{Problemstrukturen}

Die Problemstrukturen zum barrierefreien bzw. -reduzierten Wohnraum wurden dreigeteilt in die übergeordneten Strukturen der regionalen Wohnungsmärkte, in verschiedene Zielkonflikte sowie die Querschnittsthematik zur Sozialpolitik und Stadtentwicklung. Dabei sind Problemstrukturen nicht objektiv oder statisch, sondern werden von den einzelnen Akteuren im Sinne ihrer Interessen gerahmt (Böcher/Töller 2019: 35, vgl. Kapitel 4.3).

Während die Wohnungsmärkte in Sachsen-Anhalt von Alterung, Fortzug und Leerstand geprägt sind, stellt in Hessen die Wohnraumknappheit im Rhein-Main-Gebiet das zentrale wohnungspolitische Problem dar. Damit verbunden ist in Sachsen-Anhalt ein Fokus auf den Bestandsumbau, wohingegen in Hessen der Neubau stärker ins Gewicht fällt. Gleichzeitig stehen Wohnungsmarkt und -politik in beiden Bundesländern vor ähnlichen strategischen Herausforderungen: Entscheidungen haben aufgrund der entstehenden Pfadabhängigkeiten langfristige Wirkungen, obwohl das Wissen über zukünftige Wohnraumbedarfe äußerst unsicher ist. Diese Problemstruktur gilt übergeordnet für die Wohnungspolitik, aber umso mehr für den Teilbereich des barrierefreien bzw. -reduzierten Wohnraums: In welcher Form - etwa: gemeinschaftlich, Mehrgenerationen-Wohnen, allein - und wo - etwa: zentral, städtische Randlage, ländlich - wird entsprechend ausgestatteter Wohnraum zukünftig nachgefragt und wie lassen sich Angebot und Nachfrage zielsicher zusammenbringen?

Die Situation auf den Wohnungsmärkten hat Auswirkungen auf die Deutung von Zielkonflikten. Auf den angespannten Wohnungsmärkten im Rhein-Main-Gebiet artikuliert die hessische Wohnungs- und Bauwirtschaft ein klares Konfliktpotential zwischen barrierefreiem und bezahlbarem Wohnraum. Auch in Sachsen-Anhalt spielt die Frage der Bezahlbarkeit eine Rolle, allerdings stärker durch die Miet- und Kaufkraft der Nachfrageseite bedingt. Behindertenverbände indessen argumentieren hier grundsätzlich umgekehrt: In Sachsen-Anhalt seien gerade die dortigen entspannten Wohnungsmärkte in der Lage, durch das geringe Mietniveau entsprechende Zusatzbedarfe zu decken. In Hessen wiederum heißt es, dass vor allem in hochpreisigen Phasen solche Zusatzbedarfe leichter zu integrieren seien. Ohnehin wehren sich verschiedene Akteure gegen die pauschale Aussage, barrierefreie Standards im Neubau würden Mehrkosten hervorrufen. Diese umstrittene Wissenslage zur Verfügbarkeit von technischen Lösungen und ihren Kosten prägt den Untersuchungsgegenstand maßgeblich. Erschwerend kommt hinzu, dass Mehrkosten oder ein Mehraufwand in der Planung direkt ersichtlich sind, wohingegen Folgekosten wie ein Umzug in stationäre Einrichtungen, soziale Vereinsamung und Unfälle durch eine fehlende Barrierefreiheit weitgehend intransparent verbleiben. Generell ist das »Problem« von fehlendem barrierefreien bzw. -reduziertem Wohnraum insofern wenig sichtbar, als viele Menschen auch mit körperlichen oder seelischen Einschränkungen in nicht-barrierefreien Wohnungen leben, sich aber vielfach damit arrangieren.

Weitere Zielkonflikte sehen die Interviewpartner*innen in den Wohnraumbedarfen von Menschen mit unterschiedlichen Beeinträchtigungen. So benötigen etwa Menschen mit Seh- und Hörbeeinträchtigungen oder demenzieller Erkrankung andere Wohnungszuschnitte als Menschen mit Gehbeeinträchtigung. Hier bestehen 
Querverbindungen zum Erklärungsfaktor »Akteure und ihre Handlungen«, da diese Heterogenität die gemeinsame Interessenvertretung von Behindertenverbänden erschwert (vgl. Kapitel 7.3.5). Außerdem reduziert eine solche selektive Problembetroffenheit die Wahrscheinlichkeit einer breitenwirksamen politischen Adressierung des Themas (Böcher/Töller 2012: 193). Auch aus diesem Grund rahmen Behinderten- und Sozialverbände barrierefreien bzw. -reduzierten Wohnraum vielfach nicht auf Basis einzelner Zielgruppen, sondern sprechen von einem Nutzergewinn für alle Menschen und wollen damit eine breitere gesellschaftliche Akzeptanz erreichen. Dabei ist entsprechend ausgestatteter Wohnraum nicht als isoliertes Ziel zu betrachten, sondern kann im Zielkonflikt zu weiteren wohnungsbaulichen Interessen wie Energieeffizienz, Denkmal- oder Brandschutz stehen. Veränderungsinteressen für eine stärkere politische Steuerung von barrierefreiem bzw. -reduziertem Wohnraum versuchen diese Spannungsverhältnisse aufzulösen, wohingegen die Wohnungs- und Bauwirtschaft auf ein notwendiges Abwägen und bei Bedarf ein Aufweichen der barrierefreien Vorgaben besteht.

Eine große Einigkeit besteht hingegen dabei, dass es sich um ein Querschnittsthema mit Bezug zur Sozialpolitik, Stadtentwicklung und sozialen Belangen des Wohnens handelt. Die Bearbeitung von Querschnittsaufgaben steht allerdings im Konflikt zur Ressortlogik der politischen Steuerung. Sowohl bei den Institutionen als auch bei Akteuren wird diese Herausforderung immer wieder deutlich (vgl. Kapitel 7.3.4 und 7.3.5).

Prinzipiell teilen alle Akteure die Einschätzung, dass es zu wenig »bedarfsgerechten « und vor allem zugleich bezahlbaren Wohnraum gibt. Mit Roberts (2000: 1) gesprochen besteht also ein Konsens hinsichtlich der Problemdefinition, nicht hingegen in Bezug auf die Problemlösung (vgl. auch Böcher/Töller 2019: 105f.). Uneinigkeiten in der Problemlösung beginnen schon bei der Frage, was bedarfsgerechter Wohnraum ist - etwa: barrierefrei nach DIN 18040-2 oder individuell barrierereduziert angepasst - und wer zur Lösung dieses Problems zuständig ist. Hier wird barrierefreier bzw. -reduzierter Wohnraum deshalb als ein komplexes Problem mit klarer Problemdefinition und strittiger Problemlösung ausgelegt. Gleichzeitig erklärt ein Vertreter von Menschen mit Behinderungen: "Und unser Problem ist immer: Wenn etwas warten muss - die Zeit haben wir nicht. Weil, [...] wir werden rasend schnell alt. Also, ich bin jetzt 52, aber mein Zustand ist, mein Gott, so wie 72 [...]. Ich kann eben nicht warten bis 2024 [...], uns rennt die Zeit weg" (IP 23: 82ff., 128). Diese Erläuterung entspricht einem super wicked Problem, nach dem Probleme besonders drängend sind und die Zeit bei Nichthandeln immer knapper wird (Levin et al. 2012: 124). Auch die Interpretation als einfaches, komplexes oder (super) wicked Problem unterliegt damit subjektiven Einschätzungen der Akteure. Insgesamt erschweren die hier identifizierten komplexen und unsicheren Problemstrukturen eine politische Problembehandlung, weil mit ihnen das Risiko von Fehlentscheidungen wächst und dadurch ein Aufschieben politischer Entscheidungen wahrscheinlicher wird (Reiter/Töller 2014: 101; Böcher/Töller 2012: 193). 


\section{Zentrale Erkenntnisse zu »Problemstrukturen«}

Die Strukturen auf den regionalen Wohnungsmärkten beeinflussen maßgeblich die akteursspezifische Interpretation der Problemstrukturen.

Der barrierefreie bzw. -reduzierte Wohnraum ist durch die Faktoren Langfristigkeit, Unsicherheit und Zielkonflikte geprägt. Die Unsicherheit zur zukünftigen Wohnraumnachfrage und zu Mehrkosten der Barrierefreiheit wird durch die langfristigen Wirkungen wohnungspolitischer Entscheidungen noch gravierender. Zielkonflikte bestehen zwischen bezahlbarem und barrierefreiem Wohnraum, aber auch zwischen unterschiedlichen Nutzergruppen mit spezifischen Wohnbedarfen und finanziellen Handlungsspielräumen.

Es handelt sich um ein komplexes Problem mit klarer Problemdefinition (»zu wenig bedarfsgerechter Wohnraum«) bei unklarer Problemlösung (vgl. Roberts 2000: 1).

\subsubsection{Situative Aspekte}

Situative Aspekte sind kaum zu prognostizieren, können aber eigendynamisch und mit unerwarteter Stärke bestehende Policy-Ideen in ihrer Durchsetzung befördern oder umgekehrt die Aufmerksamkeit auf andere Themenbereiche lenken (vgl. Kapitel 4.4). Entsprechend unterschiedlich fällt die Rolle dieses Erklärungsfaktors in Sachsen-Anhalt und Hessen aus.

So sind in Sachsen-Anhalt mehr situative Aspekte nachzuweisen als in Hessen. Zwar wird die Bauordnungsnovellierung mit einem generellen Überarbeitungsbedarf begründet, aber gleichfalls mit einer notwendigen Harmonisierung des Landesrechts mit europäischen Vorgaben. Zudem besteht seit Herbst 2018 eine neue Debatte zur Änderung der Bauordnung, ursprünglich initiiert durch einen AfD-Antrag zur kleinen Bauvorlagenberechtigung für das Handwerk. Diesen Änderungsvorstoß machen sich im weiteren Verlauf auch Interessenvertretungen von Menschen mit Behinderungen als Möglichkeitsfenster zu eigen. Und in der Wohnraumförderung nutzt Sachsen-Anhalt die ursächlich durch den verstärkten außereuropäischen Zuzug ausgegebenen Kompensationsmittel des Bundes für die nachträgliche Aufzugsförderung. Hiermit wird einer schon seit längerem bestehenden Forderung der Wohnungs- und Bauwirtschaft Rechnung getragen. Auch erfährt der langjährige Appell nach einer hauptamtlichen Fachstelle für Barrierefreiheit Aufwind durch einen situativen Aspekt, nämlich die sogenannte EU-Webseitenrichtlinie, für die ohnehin eine entsprechende barrierefreie Beratungsinfrastruktur im Land aufgebaut werden muss.

In Hessen wiederum besteht im Bauordnungsrecht ebenfalls ein Anpassungsdruck an europarechtliche Vorgaben, hier wiegt aber die turnusmäßige Überarbeitung der Bauordnung schwerer. Und die in Sachsen-Anhalt als Möglichkeitsfenster wirkende sogenannte Flüchtlingskrise tritt in Hessen eher als Unmöglichkeitsfenster auf. Zwar wird in Reaktion darauf das Kommunalinvestitionsprogramm aufgelegt - unter ande- 
rem mit einer Förderung für Wohnraum mit barrierefreien Bestandteilen -, allerdings trifft der zusätzliche Zuzug nach Hessen auf ohnehin angespannte Wohnungsmärkte. Der wohnungspolitische Fokus auf die Bezahlbarkeit in Ballungsregionen verstärkt sich weiter und der barrierefreie bzw. -reduzierte Wohnraum wird von der politischen Agenda gespült.

Situative Aspekte verschaffen langjährigen Policy-Ideen als Möglichkeitsfenster also Aufwind oder blockieren bzw. verzögern sie als Unmöglichkeitsfenster. Analytisch verhelfen sie damit sowohl zur Erklärung von Politikwandel als auch zur Erklärung von der Beibehaltung des Status Quo. Dies verdeutlicht den dynamischen Charakter politischer Prozesse, die keinesfalls linear und orientiert an einem stärker oder schwächer werdenden Handlungsdruck erfolgen müssen (Böcher/Töller 2012: 190f.; Cairney/Zahariadis 2016: 87). Hierbei spielt es eine zentrale Rolle, inwieweit Akteure situative Aspekte zur Durchsetzung ihrer präferierten Policy-Ideen oder umgekehrt zur Ausbremsung ungewollter politischer Vorhaben nutzen können.

\section{Zentrale Erkenntnisse zu »situativen Aspekten«}

Möglichkeitsfenster für den barrierefreien bzw. -reduzierten Wohnraum liegen in Sachsen-Anhalt in europarechtlichen Vorgaben mit landespolitischem Harmonisierungsbedarf sowie im außereuropäischen Zuzug seit 2015.

In Hessen wirkt eben dieser Zuzug als Unmöglichkeitsfenster, weil er die bestehende Wohnraumknappheit im Rhein-Main-Cebiet verschärft und die Handlungsspielräume für die Barrierefreiheit damit weiter reduziert.

Situative Aspekte verschaffen bestehenden Forderungen Aufwind oder Cegenwind. Damit dienen sie den Veränderungs- bzw. Status-Quo-Interessen als Ressource.

\subsubsection{Institutionen}

Institutionen wurden in der theoretischen Vorarbeit in erstens inklusionspolitische Abkommen und Konventionen, zweitens den ministeriellen Zuschnitt im Bundesland mit Formen der positiven und negativen Koordination sowie drittens Scaling-Prozesse im föderalen Mehrebenensystem gegliedert (vgl. Kapitel 4.5). Diese institutionellen Regelungen beeinflussen die politischen Prozesse zum barrierefreien bzw. -reduzierten Wohnraum sowohl in Sachsen-Anhalt als auch in Hessen, wobei je Besonderheiten in den beiden Ländern herausgearbeitet werden können.

Inklusionspolitische Abkommen und Konventionen

Die zentrale Leitlinie der Inklusionspolitik ist die UN-Behindertenrechtskonvention. Sie bescheinigt Menschen mit Behinderungen eine gleichberechtigte Zugänglichkeit zur physischen Umwelt sowie gleiche Wahlmöglichkeiten zu einer unabhängigen Lebensführung und damit das Recht, selbst zu entscheiden, wo und mit wem sie leben (vgl. Kapitel 2.2). Insbesondere in der Argumentation zu einer stärkeren Verankerung 
von Barrierefreiheit im Bauordnungsrecht verweisen Behinderten- und Sozialverbände auf die von Deutschland im Jahr 2009 ratifizierte Konvention. In beiden Bundesländern wurden zudem Landesaktionspläne zur Umsetzung der UN-BRK formuliert, auf die sich jeweils stärker die politisch-administrativen Akteure berufen. Womöglich zeigt sich hier, dass die UN-BRK selbst den umfassenderen normativen Anspruch besitzt und Aktionspläne bereits verschiedenen Interpretationen und Einschränkungen unterliegen. Außerparlamentarische Interessen bevorzugen folglich einen Verweis auf den übergeordneten institutionellen Rahmen.

Ebenfalls wichtig sind die Behindertengleichstellungsgesetze, da sie eine konkrete Definition von Barrierefreiheit liefern und diese Definition wiederum Eingang in das Bauordnungsrecht findet. Die Debatte zur "Auffindbarkeit« als Kriterium für Barrierefreiheit, welche im novellierten HessBGG 2019 integriert wurde, nicht aber in der kurz zuvor verabschiedeten hessischen Bauordnung, offenbart dabei die verzögerte Wirkung, mit der inklusionspolitische Belange Einzug in andere Politikbereiche finden. Auch wenn Behinderten- und Sozialverbände auf das 2016er Bundes-BGG und die darin enthaltene "Auffindbarkeit« verweisen, bedarf es offenbar zunächst einer Überführung in das einschlägige Landesgesetz (vgl. Kapitel 7.2.5). Trotz dieser Zeitverzögerung zeigt sich hier exemplarisch, dass auch institutionelle Regelungen Wandel hervorrufen können. Veränderungsinteressen nutzen die UN-BRK, Landesaktionspläne und Behindertengleichstellungsgesetze zur Unterfütterung ihrer Positionen. Darin offenbart sich wiederum das interdependente Verhältnis von Institutionen und Akteuren: Gesellschaftlich veränderte Normvorstellungen werden institutionalisiert und beeinflussen daraufhin weiterführende politische Prozesse.

Im Bundesland: Ministerieller Zuschnitt und Politikintegration

Der Erfolg einer Überführung inklusionspolitischer Belange in weitere Politikfelder hängt maßgeblich mit dem Ressortzuschnitt zusammen, gibt dieser doch Aufschluss über die politische Prioritätensetzung, Machtverteilung und Kooperationspotentiale zwischen den Ressorts (Linhart/Windwehr 2012: 579f.; Leunig 2012: 188; Korte 2010: 29). Tabelle 10 zeigt in Sachsen-Anhalt im Untersuchungszeitraum stabile Zuständigkeiten für Bauen/Wohnen und Soziales. Das Bauordnungsrecht und die Wohnraumförderung liegen im CDU-geführten Verkehrsministerium, das Sozialministerium ist SPD-geführt. Der hessische Ressortzuschnitt und die parteipolitische Färbung sind unbeständiger. Von 2014 bis 2019 waren Bauordnungsrecht und Wohnraumförderung auf die grünen Wirtschafts- und Umweltministerien aufgeteilt, während das Sozialministerium CDU-Minister Grüttner unterstellt war. Seit 2019 liegen Bauordnungsrecht und Wohnraumförderung beim grünen Wirtschaftsminister und auch das Sozialministerium ist unter grüner Führung. Eine gleiche parteipolitische Färbung könnte eine interministerielle Kooperation erleichtern, was sich aber erst im weiteren Verlauf überprüfen lässt. 
Tabelle 10: Ressortzuschnitte in Sachsen-Anhalt und Hessen (eigene Darstellung)

\begin{tabular}{|c|c|c|c|}
\hline & \multirow{2}{*}{ Regierung } & \multicolumn{2}{|c|}{ Ministerielle Zuständigkeit } \\
\hline & & Bauen/Wohnen & Soziales \\
\hline \multirow[t]{2}{*}{$\begin{array}{l}\text { Sachsen- } \\
\text { Anhalt }\end{array}$} & $\begin{array}{l}\text { 2011-2016: } \\
\text { Haseloff I, } \\
\text { schwarz-rote Koalition }\end{array}$ & \multirow[t]{2}{*}{$\begin{array}{l}\text { Verkehrsministerium } \\
\text { (CDU) }\end{array}$} & \multirow[t]{2}{*}{$\begin{array}{l}\text { Sozialministerium } \\
\text { (SPD) }\end{array}$} \\
\hline & $\begin{array}{l}\text { 2016-2021: } \\
\text { Haseloff II, } \\
\text { schwarz-rot-grüne Koalition }\end{array}$ & & \\
\hline \multirow[t]{2}{*}{ Hessen } & $\begin{array}{l}\text { 2014-2019: Bouffier II, } \\
\text { schwarz-grüne Koalition }\end{array}$ & $\begin{array}{l}\text { Wirtschaftsministerium } \\
\text { zum Bauordnungsrecht, } \\
\text { Umweltministerium zur } \\
\text { Wohnraumförderung (je } \\
\text { Bündnis 9o/Die Grünen) }\end{array}$ & $\begin{array}{l}\text { Sozialministerium } \\
\text { (CDU) }\end{array}$ \\
\hline & $\begin{array}{l}\text { 2019-2024: } \\
\text { Bouffier III, } \\
\text { schwarz-grüne Koalition }\end{array}$ & $\begin{array}{l}\text { Wirtschaftsministerium } \\
\text { (Bündnis 90/Die Grünen) }\end{array}$ & $\begin{array}{l}\text { Sozialministerium } \\
\text { (Bündnis 9o/ } \\
\text { Die Grünen) }\end{array}$ \\
\hline
\end{tabular}

Generell dominiert in beiden Bundesländern die negative Koordination der Ressortlogik das ministerielle Handeln. Wie die vorherigen Ausführungen zu inklusionspolitischen Abkommen zeigen, bestehen zwar durchaus Versuche der Politikintegration, die sich etwa im hessischen Normprüfleitfaden, in den eingerichteten Referaten zur Umsetzung der UN-BRK und in der Funktion des/der Landesbeauftragten für Menschen mit Behinderungen manifestieren. Allerdings bestehen in den untersuchten politischen Prozessen deutliche Unterschiede in der ressortübergreifenden Wirksamkeit dieser Formate. So treten der sachsen-anhaltische Behindertenbeauftragte und die ihm unterstellten Gremien als aktive Veränderungsinteressen auf, wohingegen die Behindertenbeauftragte in Hessen beim barrierefreien bzw. -reduzierten Wohnraum nicht öffentlichkeitswirksam aufscheint. Auch das für die UN-BRK zuständige Referat sowie der Landesaktionsplan werden von hessischen Behindertenverbänden nicht als Verbündete zur gemeinsamen Interessenvertretung angesehen: »Und der Hessische Aktionsplan ist alles andere als progressiv« (IP 46: 110).

Gleichzeitig sind solche Einordnungen immer Momentaufnahmen und letztlich von den jeweiligen Individuen abhängig, die die entsprechenden Ämter bekleiden. Diesbezüglich verändern sich in Hessen in den Jahren 2019/20 verschiedene Strukturen, indem nicht nur eine Neubesetzung des Amts der Behindertenbeauftragten stattfindet, sondern die neue Amtsträgerin zukünftig hauptamtlich dieser Tätigkeit nachgehen wird. Zudem sollen die inklusionspolitischen Maßnahmen im Sozialministerium gebündelt werden. Und auch in Sachsen-Anhalt bestand zum Herbst 2019 insofern ein Wechsel, als der seit 2005 tätige Behindertenbeauftragte durch einen Nachfolger abgelöst worden ist. Solche personellen Veränderungen können neue Dynamiken auslösen, die das interdependente Zusammenspiel von Institutionen und in ihnen handelnden Akteuren unterstreichen. 
Generell ist das Disability Mainstreaming aus dem Sozialressort in den Bereich Bauen/Wohnen eine Herausforderung. So heißt es aus Sachsen-Anhalt, der Beauftragte würde entsprechende Gesetzesvorlagen vielfach erst spät zur Durchsicht bekommen. Rudzio (2019: 237) spricht insgesamt skeptisch von einer inflationären Einrichtung solcher Ämter, was der »symbolischen Repräsentation von Problemgruppen« diene. Zur breiten und verbindlichen Verankerung der Behindertenbeauftragten regen entsprechend sowohl in Sachsen-Anhalt als auch in Hessen Veränderungsinteressen an, diese Funktion aus dem Sozialressort heraus in der Staatskanzlei anzugliedern. Hier zeigt sich deutlich die Herausforderung von Querschnittsaufgaben, die bereits unter den Problemstrukturen herausgearbeitet worden ist (vgl. auch Böcher/Töller 2019: 73, 103f.). Damit gehen nicht nur Koordinationsbedarfe einher, sondern auch Akteurkonstellationen, »die vielschichtig und fragil bis schlicht unübersichtlich sein können « (Heinelt 2003: 251). Hier scheint bereits eine weitere Querverbindung auf, die unter »Akteure und ihre Handlungen« $\mathrm{zu}$ vertiefen ist (vgl. Kapitel 7.3.5).

Im föderalen Mehrebenensystem: Up-, Down- und Through-Scaling

Trotz der ordinären Landeszuständigkeiten im Bauordnungsrecht und in der Wohnraumförderung können sich die Länder untereinander sowie mit dem Bund und ihren Kommunen koordinieren und in ihren politischen Prozessen gegenseitig beeinflussen (vgl. Kapitel 4.5.3).

Beim Through-Scaling beeinflussen ministerielle oder parteipolitische länderübergreifende Arbeitsgruppen die Politikgestaltung, etwa über die ARGEBAU-Gremien. Die empirischen Ergebnisse bescheinigen diesen Formaten jedoch eine ambivalente, begrenzte Wirkung. So gilt die Musterbauordnung zwar als zentrale Leitlinie und beeinflusst das Bauordnungsrecht insbesondere in Sachsen-Anhalt maßgeblich, die Wohnraumförderung hingegen ist sowohl in Sachsen-Anhalt als auch in Hessen stärker durch landesspezifische Strukturen geprägt. Statt dem Austausch in formalisierten Formaten würde - wenn überhaupt - anlassbezogen bei konkreten Vorhaben in anderen Bundesländern nach ähnlichen Förderprogrammen gesucht. Dann dienen Länder mit vergleichbaren Wohnungsmärkten oder Haushaltslagen als Orientierungspunkt. In Hessen kommt hinzu, dass verschiedene Verbände landesübergreifend organisiert sind etwa der VdK Hessen-Thüringen oder der VdW Südwest. Diese außerparlamentarischen Interessen verweisen auf Policy-Ausprägungen in anderen Bundesländern, sofern diese ihren Präferenzen entsprechen.

Das Up- und Down-Scaling von bundespolitischen oder kommunalen Maßnahmen beeinflusst die politischen Prozesse in beiden Bundesländern unterschiedlich stark. In Sachsen-Anhalt sind die Kommunen in der Steuerung von barrierefreiem bzw. -reduziertem Wohnraum zurückhaltend, womit wenig Raum für Up-Scaling besteht. Die sachsen-anhaltische Förderlandschaft richtet sich allerdings am KfW-Programm »Altersgerecht Umbauen« aus und auch die Fachstelle Barrierefreiheit findet auf der Bundesebene eine Referenz. Hessen wiederum orientiert sich weniger an bundespolitischen Aktivitäten und setzt stärker auf eine eigene Politikgestaltung, was dem Selbstverständnis eines finanzstarken, selbstbewussten Gliedstaats entspricht (Carstensen 2017: 117; Schroeder/Neumann 2016b: 1ff.). Und auch die hessischen Kommunen entwi- 
ckeln einschlägige Beratungsformate oder Förderprogramme. Diese Aktivitäten finden jedoch eher parallel zu den landespolitischen Maßnahmen statt und beeinflussen diese nicht in Form eines vertikalen Up-Scaling.

\section{Zentrale Erkenntnisse zu »Institutionen «}

Inklusionspolitische Abkommen erzeugen normativen Handlungsdruck auf wohnungspolitische Debatten und können damit Politikwandel hervorrufen.

Zwischen Sozial- und Bauressort dominiert die negative Koordination, während Versuche der Politikintegration in der praktischen Umsetzung an ihre Grenzen stoßen.

Im föderalen Mehrebenensystem finden sich Formen des horizontalen Through-Scaling (vor allem: ARGEBAU-Musterbauordnung) und des vertikalen Down-Scaling (vor allem: KfW-Programm »Altersgerecht Umbauen«), aber insgesamt prägen die Strukturen im Bundesland die politischen Prozesse stärker als Anreize von außerhalb.

\subsubsection{Akteure und ihre Handlungen}

Der letzte AEP-Erklärungsfaktor ist durch die handlungstheoretische Ausrichtung der AEP-Heuristik zugleich der wichtigste. Mithilfe der Kategorien »Interessen«, »Ressourcen« und »Strategien« wurde die Rolle der verschiedenen Akteursgruppen in den politischen Prozessen zum barrierefreien bzw. -reduzierten Wohnraum analysiert (vgl. Kapitel 4.6).

Übergeordnete Strukturierung der Akteure

Die außerparlamentarischen Interessen wurden in die Angebots- und Nachfrageseite nach Wohnraum unterteilt. Die Angebotsseite aus Wohnungs- und Bauwirtschaft konzentriert sich in Sachsen-Anhalt auf den Bestandsumbau, während die hessischen Äquivalente sich stärker in die politische Steuerung des Neubaus einbringen. Dies ist den Strukturen der regionalen Wohnungsmärkte geschuldet und erklärt zugleich, warum sich in Hessen auch Architekt*innen, Bauingenieur*innen sowie das Bündnis »Allianz für Wohnen« engagieren. Die Nachfrageseite nach barrierefreiem bzw. -reduziertem Wohnraum besteht in beiden Ländern vorwiegend aus Vertretungen von Menschen mit Behinderungen. In Sachsen-Anhalt sind auch korporatistische Formate wie der Behindertenbeauftragte und -beirat aktiv, in Hessen wird zudem der Sozialverband VdK als Veränderungsinteresse ausgemacht. Die ursprünglich vermutete Beteiligung von Seniorenvertretungen findet sich nicht, hier dominieren offensichtlich andere Themen und eine Beschäftigung mit »Alter und Krankheit« beginnt erst mit eigener Betroffenheit. Auch Mieterverbände als einschlägige wohnungspolitische Akteure treten beim barrierefreien bzw. -reduzierten Wohnraum nicht als aktive Player auf. Sie setzen sich mutmaßlich stärker für allgemeinere Mieterinteressen ein.

Die parteipolitischen Akteure wurden entlang ihrer Motive zum Policy- und Votebzw. Office-Seeking analysiert. Im Untersuchungszeitraum regiert in Sachsen-Anhalt zunächst eine schwarz-rote, dann eine schwarz-rot-grüne Koalition. In Hessen wird die 
schwarz-grüne Koalition im Jahr 2018 noch einmal im Amt bestätigt. Ausgeprägte parteipolitische Differenzen zeigen sich auf Ebene der politischen Zielsetzung nicht. Vielmehr ist barrierefreier bzw. -reduzierter Wohnraum ein wenig salientes und zugleich konsensuales Thema, das alle Parteien zumindest als Zielvorgabe adressieren (vgl. Ruß 2014: 354). Bei einzelnen politischen Vorhaben oder Policy-Ausprägungen treten allerdings die Oppositionskräfte DIE LINKE in Sachsen-Anhalt sowie die hessische SPD als Forder*innen auf. Zudem ist in der sachsen-anhaltischen Regierung deutlich die SPD als diejenige Regierungsfraktion auszumachen, die sich für entsprechende politische Vorhaben einsetzt.

In der Ministerialverwaltung interessierten die jeweiligen Fachabteilungen in den für Bauen/Wohnen und Soziales zuständigen Ministerien. Die theoretischen Annahmen zu eigenen wert- oder zweckrationalen Interessen sowie zum bürokratischen Agenda-Setting und der strategischen Interaktion lassen sich dabei nur anhand konkreter politischer Vorhaben überprüfen. Inwieweit führen Verwaltungsakteure als kritisch-wissensbasierte Berater*innen die politischen Zielvorgaben ihrer Prinzipale aus, inwieweit verfolgen im Sinne einer hybriden Politisierung eigene Interessen?

\section{Bauordnungsrecht}

Das Bauordnungsrecht ist das zentrale regulative Instrumentarium zum barrierefreien bzw. -reduzierten Wohnraum. Die Akteursanalysen konzentrierten sich dabei jeweils auf die letzte große Bauordnungsnovellierung: In Sachsen-Anhalt im Jahr 2013 und in Hessen in den Jahren 2017/18. Gleichzeitig werden im Sinne eines inkrementellen Politikstils auch langfristige Einflussnahmen nachgewiesen, die über die eigentlichen Novellierungsprozesse hinausgehen.

Das Engagement außerparlamentarischer Interessen unterscheidet sich in den beiden Ländern grundlegend. Bedingt durch das stärkere Gewicht von Neubauvorhaben ist die hessische Wohnungs- und Bauwirtschaft aktiver als ihr sachsen-anhaltisches Pendant. In Sachsen-Anhalt beschränkt zudem die strikte Orientierung an der Musterbauordnung landesspezifische Änderungspotentiale. Das barrierefreie Bauen wird von der dortigen Angebotsseite von Wohnraum ohnehin wenig thematisiert - sie trägt die entsprechenden Gesetzesänderungen weitgehend mit. Behindertenvertretungen fordern hingegen Nachbesserungen ein und nutzen bereits seit den 1990er Jahren verschiedene Formate der inkrementellen Einflussnahme. In den parlamentarischen Raum hinein bestehen Kooperationen zu DIE LINKE, die sich in verschiedenen Kleinen Anfragen niederschlagen. Diese Lobbying-Strategien sind in ihrer Wirkung bislang aber eher verhalten.

In Hessen ist nicht nur die Nachfrageseite nach Wohnraum in Form von unterschiedlichen Behindertenverbänden und dem VdK aktiv, auch eine breite Koalition der Angebotsseite von Wohnraum bezieht Position zur baulichen Barrierefreiheit (vgl. Abbildung 30). Mit dem Argument der Kostensteigerung fordert diese Koalition eine Reduzierung der barrierefreien Vorgaben. Der Gesetzentwurf vom Winter 2017 enthält diesbezüglich bereits verschiedene Änderungen, wobei die 20-Prozent-Regelung sowie die Streichung der rollstuhlgerechten Zugänglichkeit besonders umstritten sind. Diese Aspekte werden von der Nachfrageseite als Verschlechterung kritisiert, jedoch 
erweist sich der Gesetzentwurf als weitestgehend stabil und verdeutlicht damit, wie wichtig eine frühzeitige Einflussnahme ist. Während die Angebotsseite bereits im Sommer 2017 ihre Positionen kundtut, ist die Nachfrageseite zwar ebenfalls vorab mit dem Wirtschaftsministerium im Gespräch - damals existiert die umstrittene 20-ProzentRegelung jedoch noch gar nicht. Die richtigen Zugänge und Zeitpunkte erscheinen hier also wesentlich. Um seine Unzufriedenheit mit der beschlossenen Bauordnungsnovelle kundzutun, initiiert der VdK im Nachhinein eine Online-Petition und nutzt damit eine Form des offensiven Outside-Lobbying. Im Frühjahr 2020 war dazu ein Runder Tisch mit dem Wirtschaftsministerium geplant, hier wirkt die Corona-Pandemie jedoch als Unmöglichkeitsfenster und verzögert die politischen Prozesse auf unbestimmte Zeit.

Die Fraktionen im sachsen-anhaltischen und hessischen Landtag unterscheiden sich in den Bauordnungsnovellierungen in ihrer Rhetorik nicht grundlegend voneinander. In beiden Ländern bescheinigen die für Bauen/Wohnen zuständigen Minister und die wohnungsbaupolitischen Sprecher*innen der Regierungsfraktionen eine Verbesserung der Barrierefreiheit. Diese Akteure betonen die Fortschritte im Vergleich zur je vorherigen Bauordnung. Umgekehrt kritisieren verschiedene Oppositionsparteien die geplanten Regelungen - in Sachsen-Anhalt DIE LINKE, in Hessen insbesondere die SPD. Die Online-Petition des VdK Hessen-Thüringen wird dann auch aktiv durch die wohnungsbaupolitische Sprecherin der SPD unterstützt. Einbringungen der sozialpolitischen Sprecher*innen, wie sie das Disability Mainstreaming nahelegen würde, gibt es nicht.

Die Fachabteilungen im sachsen-anhaltischen Verkehrs- und im hessischen Wirtschaftsministerium sind für die Gesetzesvorbereitung im Bauordnungsrecht zuständig. Insbesondere in Sachsen-Anhalt wird das mit dieser Zuständigkeit einhergehende Potential zur strategischen Interaktion aber durch die enge Anlehnung an die Musterbauordnung geschmälert. Auch wenn die hessische Landesregierung ebenfalls auf das Bundesmuster verweist, zeigt der dortige Gesetzentwurf doch größere landesspezifische Handlungsspielräume auf. Hier entscheidet die Ministerialverwaltung, welchen außerparlamentarischen Interessen sie wie viel Gehör schenkt. Nachträgliche Änderungen am Gesetzentwurf durch parlamentarische Initiativen sind, wie gezeigt, eher selten. Formen des Disability Mainstreaming über Akteure des Sozialressorts bestehen lediglich eingeschränkt. In Hessen ist in diesem Kontext der Normprüfleitfaden interessant, welcher dem Gesetzentwurf eine Konformität mit der UN-Behindertenrechtskonvention bescheinigt. Es verbleibt jedoch unklar, ob die Oberste Bauaufsicht oder das Referat zur Umsetzung der UN-BRK diese Beurteilung vorgenommen hatte und Behindertenverbände zweifeln die Konformität des Gesetzesentwurfes an. Ebenfalls in Hessen ist ein neuer Bauvorlagenerlass (»Barrierefrei-Konzept«) von Interesse. Dieser wird gemeinsam mit der novellierten Bauordnung eingeführt - und das völlig unterhalb des politischen Radars. Hier ist die Ministerialverwaltung die treibende Kraft im politisch-administrativen System und reagiert eigenständig auf eine Forderung der Nachfrageseite nach Wohnraum. 
Wohnraumförderung

Die Wohnraumförderung basiert in Hessen auf einem Wohnraumfördergesetz, darüber hinaus formulieren die Koalitionsverträge in Sachsen-Anhalt und Hessen entsprechende politische Vorhaben. Abgesehen von solchen übergeordneten Leitlinien aus der Politik ist die Förderkulisse über den Erlass von Richtlinien organisiert und damit weitgehend »Verwaltungshandeln« (IP 53: 29).

Hier tritt die Wohnungs- und Bauwirtschaft als Veränderungsinteresse auf, da sie als Adressatin der Förderprogramme ein Eigeninteresse an attraktiven Förderkonditionen hat. Dabei kommt, wie bereits gezeigt, in Hessen Neubauvorhaben und vor allem dem schnellen, bezahlbaren Wohnraum ein stärkeres Gewicht zu, wohingegen der barrierereduzierende Bestandsumbau in Sachsen-Anhalt eine Priorität darstellt. So geht das AUFZUGS-Programm dann auch maßgeblich auf Initiativen der sachsen-anhaltischen Wohnungswirtschaft zurück. Beiderorts nutzt die Angebotsseite von Wohnraum zur Durchsetzung ihrer Interessen verschiedene Formen der inkrementellen Einflussnahme, wobei die je zuständigen ministeriellen Fachabteilungen bereits proaktiv auf die Wohnungs- und Bauwirtschaft zukommen. In diesem Kontext ist in Hessen die Allianz für Wohnen eine zentrale, die langfristige Förderkulisse mitgestaltende Instanz. Anlassbezogene Richtlinien-Überarbeitungen finden hingegen in beiden Bundesländern im direkten Dialog mit einzelnen Akteuren der Wohnungs- und Bauwirtschaft statt. Die Nachfrageseite nach barrierefreiem bzw. -reduziertem Wohnraum ist in diese Prozesse nicht involviert, auch wenn insbesondere in Sachsen-Anhalt verschiedene Interessenvertretungen von Menschen mit Behinderungen versuchen, die Förderkulisse nach ihren Gunsten zu beeinflussen.

Die Landtage sind in die konkrete Ausgestaltung der Förderkonditionen regulär nicht eingebunden. Generell ist eine ökonomische Anreizförderung wenig umstritten und wird von allen parteipolitischen Akteuren befürwortet. Insbesondere in bestehenden Förderprogrammen, die als Instrumenten-Alternative nach Typ 1 sukzessive in ihren Ausprägungen an neue Bedarfe angepasst werden, ist die Einflussnahme der Fraktionen beschränkt und die Aufmerksamkeit politischer Akteure richtet sich vielmehr auf kontroverse oder öffentlichkeitswirksame Vorhaben. Entsprechend kommt der Einrichtung neuer Förderprogramme mehr politisches Gewicht zu, wie sich exemplarisch am AUFZUGS-Programm in Sachsen-Anhalt zeigt. Hier erhöhen die SPD-initiierten Debatten letztlich den Handlungsdruck auf die ministeriellen Fachabteilungen zur Umsetzung der Koalitionsvereinbarungen. Über die Notwendigkeit von bezahlbarem und altersgerechtem Wohnraum forciert die SPD als Koalitionspartnerin das so prominent diskutierte und genutzte AUFZUGS-Programm - und das, obwohl sie gar nicht das zuständige Verkehrsministerium hält.

Bei der Ausgestaltung der Wohnraumförderung verfügen die ministeriellen Fachabteilungen über ein großes Potential zur strategischen Interaktion. In beiden Bundesländern findet dieser Prozess in enger Kooperation mit der örtlichen Landesförderbank und der Wohnungs- und Bauwirtschaft statt. In Hessen etwa geschieht dies über nichtöffentliche Anhörungen, ergänzt um nachträgliche bilaterale Austausche,

»wo Sie tage-, wochenlang nur telefonieren, weil, dann sagt der Verband A wieder: ১Wir möchten dieses Wort hier geändert haben<, dann müssen wir überlegen: >Welche 
Verbände würden das nicht mittragen?«, müssen da wieder anrufen und sagen: >Das und das Wort wird jetzt geändert`-das ist sehr, sehr kleinteilig« (IP 57: 37).

Dieses Zitat zeigt die strategische Relevanz der Ministerialverwaltung: Sie entscheidet letztlich, welche außerparlamentarischen Interessen sie berücksichtigt und damit auch, welche Interessen in der Förderkulisse integriert werden. Da die Wohnungswirtschaft in Sachsen-Anhalt einen eigenen Schwerpunkt auf barrierereduzierte Anpassungsmaßnahmen legt, schaffen es solche Zusatzbedarfe hier leichter in die Wohnraumförderung - formuliert über die Angebotsseite von Wohnraum und erfolgreich über die Ministerialverwaltung in die Richtlinien getragen.

Wohnberatungen zur Wohnraumanpassung bzw. Fachstellen zur Barrierefreiheit

Die Wohnberatung zur Wohnraumanpassung hat keine feste Zuständigkeit im föderalen Mehrebenensystem, sodass die Bundesländer sich in sehr unterschiedlichem Maße engagieren. Weder in Sachsen-Anhalt noch in Hessen gibt es hierzu aktive politische Prozesse. Die seit 2002 bestehende Hessische Fachstelle für Wohnberatung weist eine stabile Pfadabhängigkeit auf und in Sachsen-Anhalt übernimmt mit Prävention im Alter ein eingetragener Verein einige Ausbildungsleistungen. Es können keine Akteure nachgewiesen werden, die sich aktiv für eine Veränderung der gegenwärtigen politischen (Nicht-)Steuerung engagieren. Mutmaßlich behindern hier die nicht geklärten föderalen Zuständigkeiten eine festere politische Verankerung.

Als alternative Form der informationellen Steuerung wird sowohl in Sachsen-Anhalt als auch in Hessen die Einrichtung einer sogenannten Fach- bzw. Kompetenzstelle Barrierefreiheit diskutiert. Während eine solche Stelle in Sachsen-Anhalt im Frühjahr 2020 ihre Arbeit aufnimmt, halten die hessischen Prozesse dazu im selben Zeitraum noch an. Eine zentrale, bündelnde Instanz entspricht dem Disability Mainstreaming und hätte je nach ihrer Ausrichtung Auswirkungen auch auf das barrierefreie bzw. -reduzierte Wohnen. Die Verantwortung für eine solche Struktur liegt in beiden Bundesländern im Sozialressort und wird von Behindertenverbänden eingefordert. Dieses Lobbying zieht sich über mehrere Jahre und wird über die Landesaktionspläne zur Umsetzung der UN-BRK gestützt. Im parlamentarischen Raum setzen sich in Sachsen-Anhalt die Koalitionsfraktion SPD und die oppositionelle DIE LINKE aktiv dafür ein, während in Hessen Bündnis 90/Die Grünen diese Forderung in den Koalitionsvertrag des Jahres 2019 verhandeln. Die jeweiligen Sozialministerien scheinen dabei nicht als aktive Veränderungsinteressen auf und werden in Sachsen-Anhalt erst durch einen Landtagsbeschluss zum Handeln bewegt. Letztlich verhilft der Policy hier die Verabschiedung einer EU-Richtlinie zum barrierefreien Internet Aufwind zur Durchsetzung.

Übergeordnete Ressourcen und Strategien

Akteuren stehen zur Durchsetzung ihrer Interessen unterschiedliche Ressourcen zur Verfügung und sie nutzen in Abhängigkeit davon unterschiedliche Strategien. Für die außerparlamentarischen Interessen lässt sich eine stärkere Durchsetzungskraft der Angebotsseite von Wohnraum nachweisen, da diese als Leistungserbringerin über eine strukturelle Macht verfügt und zudem vom politisch-administrativen System als Expertin des Wohnungsbaus wahrgenommen wird. Auch wenn »die Angebotsseite« sich 
aus privaten Kleinvermieter*innen sowie unterschiedlich organisierten Wohnungsund Bauunternehmen zusammensetzt, gelingt diesen Akteuren bei der hessischen Bauordnungsnovellierung doch eine übergeordnete Koalition. Insgesamt dominiert dabei die institutionalisierte Wohnungs- und Bauwirtschaft mit einem Schwerpunkt auf den Mietwohnraum.

Schwieriger verhält es sich bei den Ressourcen der Nachfrageseite. Einschlägige wohnungspolitische Akteure wären hier Interessenvertretungen von Mieter*innen, die aber - wie gezeigt - keinen Schwerpunkt auf barrierefreien bzw. -reduzierten Wohnraum legen. Spezialinteressen von Menschen mit Behinderungen werden hingegen vom politisch-administrativen System nicht als ebenbürtige wohnungspolitische Interessen betrachtet. Vielfach arbeiten diese Verbände im Ehrenamt und vertreten ihre Forderungen im Sinne eines Disability Mainstreamings in zahlreichen Politikbereichen. Sie sind damit weniger spezialisiert und müssen sich breiter aufstellen. Erschwerend kommt die Zersplitterung der Partikularinteressen einzelner Behindertenverbände hinzu, die eine gemeinsame Interessenvertretung "mit einer Stimme« erschwert. Die geringe Salienz des barrierefreien bzw. -reduzierten Wohnraums begrenzt außerdem die Potentiale der gesellschaftlichen Macht, da wenig öffentlichkeitswirksame Themen unter dem Gesichtspunkt des Office-Seeking nicht wahlentscheidend sind. Allerdings zeigt die Online-Petition des VdK Hessen-Thüringen, dass die Medialisierung als Kampagnenformat bislang schwachen Interessen durchaus eine stärkere Stimme verschaffen kann (vgl. von Winter 2007: 220ff.; Baruth/Schnapp 2015: 255; Töller/Böcher 2017: 552f.). Zudem verfügt die Nachfrageseite in Sachsen-Anhalt und Hessen über institutionelle Machtressourcen durch korporatistische Strukturen wie Behindertenbeauftragte und -beiräte. Die Konsequenz ist eine Mischform aus pluralistischen und korporatistischen Interessenvertretungen, die insbesondere in Sachsen-Anhalt zu einer lauten Stimme der Behindertenvertretungen führt - wenn auch, wie gezeigt, mit bislang nur mäßigem Erfolg in der letztlichen Interessendurchsetzung.

Ebenfalls als korporatistische Struktur gilt die hessische Allianz für Wohnen. Diese zielt indessen auf allgemeinere wohnungspolitische Strategien ab und ist beim barrierefreien bzw. -reduzierten Wohnraum wenig präsent. Auch hier zeigt sich die Schwierigkeit der Politikintegration: Spezialinteressen aus anderen Politikfeldern sind in einschlägigen Bündnisformaten weniger stark vertreten und haben es damit in ihrer Interessendurchsetzung schwerer. Die Anzahl der zu beteiligenden Akteure muss zur Handhabbarkeit zwangsweise begrenzt bleiben, womit die klassischen wohnungspolitischen Akteure gegenüber Partikularinteressen über einen Ressourcenvorsprung verfügen (vgl. von Winter/Willems 2009: 24).

Anders als die Ressourcen unterscheiden sich die genutzten Strategien zwischen Angebots- und Nachfrageseite nicht sonderlich stark. In beiden Bundesländern wird die Relevanz einer langfristigen und frühzeitigen Einbindung bestätigt. Sowohl Wohnungs- und Bauwirtschaft als auch Behinderten- und Sozialverbände sehen parteipolitische sowie ministerielle Akteure als wichtige Lobby-Adressat*innen an. Dabei ist die Umsetzung eines Policy-Wandels immer schwieriger als eine Beibehaltung des Status Quo - sei es eine stärkere Verankerung der Barrierefreiheit im Bauordnungsrecht oder die Einrichtung eines neuen Förderprogramms oder einer Fach-/Kompetenzstelle. Bezüglich der Strategien unterscheiden sich lediglich die 
Zugänge und das Gehör im politisch-administrativen System. So ließ sich nachweisen, wie einschlägige wohnungsmarktliche Akteure frühzeitig und eigeninitiativ von der Ministerialverwaltung in die Politikformulierung eingebunden werden. Die Nachfrageseite hingegen muss diese Beteiligung stärker einfordern und wählt im Bedarfsfall sogar Formen des Outside-Lobbying, wie die Online-Petition des VdK im Nachgang zur hessischen Bauordnungsnovelle zeigt.

Die parteipolitischen Akteure unterscheiden sich in ihrer Ressourcenausstattung am stärksten entlang der Scheidelinie »Regierungsverantwortung - Opposition«. Hier gelten die Koalitionsverträge als klare Leitlinie der jeweiligen Landesregierung, die im Verlauf der Legislaturperiode Handlungsdruck erzeugt. Unter den Handlungsbeschränkungen des Koalitionszwangs, von Pfadabhängigkeiten und der jeweiligen Haushaltslage legen die Koalitionsverträge mittelfristige Projekte fest und gelten als Bewertungsgrundlage der Landesregierung. Hier zeigt sich ebenfalls ein inkrementeller Politikstil, der die weit verbreitete Annahme der kurzlebigen, durch aktuelle Stimmungen geleiteten Politik zumindest für das politische Alltagsgeschäft widerlegt. Den Regierungsfraktionen kann dabei insbesondere zu den Ministerien derselben parteipolitischen Färbung ein besserer Zugang bescheinigt werden, aber insgesamt sind alle Regierungspartner*innen frühzeitiger als die oppositionellen Akteure in politische Vorhaben eingebunden. In Hessen zeigt sich dies exemplarisch an der Allianz für Wohnen und in Sachsen-Anhalt verdeutlicht das AUFZUGS-Programm, dass die SPD als Koalitionspartnerin ein Projekt der Wohnraumförderung durchsetzen kann, auch wenn sie nicht das dort CDU-geführte, zuständige Verkehrsministerium hält. Die Opposition ist in ihrer Ressourcenausstattung und Strategiewahl naturgemäß eingeschränkter. Gleichzeitig ließ sich nachweisen, dass insbesondere die sachsenanhaltische DIE LINKE und die hessische SPD über Bündnisse mit außerparlamentarischen Interessen sowie über parlamentarische Initiativen Handlungsdruck auf die jeweiligen Regierungen erzeugen. Gerade bei der Fachstelle für Barrierefreiheit in Sachsen-Anhalt ist dies ganz deutlich zu beobachten, aber auch bei der Bauordnungsnovellierung in Hessen. Derartige Anstrengungen des oppositionellen Agenda-Settings sind nicht zu unterschätzen, auch wenn die Wirkung vielfach erst zeitverzögert eintritt.

Der Ministerialverwaltung wurden eine langjährige Expertise im jeweiligen Regelungsfeld sowie damit verbunden stabile Netzwerke in parlamentarische und außerparlamentarische Kreise zugeschrieben. Diese Annahmen sind weitgehend zu bestätigen und kommen vor allem darin zum Ausdruck, dass die ministeriellen Fachabteilungen deutlich mehr Zeit in einzelne Gesetzesvorhaben oder Richtlinien einbringen können, als dies den parteipolitischen Akteuren möglich ist. Daraus abgeleitet stellt sich die Frage, inwieweit Akteure der Ministerialverwaltung diesen Ressourcenvorsprung zur Durchsetzung eigener wert- oder zweckrationaler Interessen nutzen oder aber stärker im Sinne einer kritischen Loyalität die teils vagen politischen Zielvorgaben ihrer Prinzipale umsetzen. Eine eigene, wertgeleitete Politikgestaltung lässt sich weder in Sachsen-Anhalt noch in Hessen nachweisen. Vielmehr kommt das Agenda-Setting in Form von Koalitionsvereinbarungen oder nachgelagert durch Landtagsbeschlüsse aus den Landesregierungen. Insbesondere in Sachsen-Anhalt sind die Akteure aus dem Sozial- und Verkehrsministerium wenig eigeninitiativ, was teils durch die personelle 
Situation in den Ministerien begründet wird, aber auch auf die Trägheit ministerieller Prozesse hindeuten könnte, in denen sich die ministeriellen Akteure zweckrational stärker am Status Quo und weniger an Veränderungsprojekten ausrichten. Außerdem kommt hier ihre begrenzte Strategiefähigkeit zum Tragen, nach der ministerielle Akteure funktionsbedingt stärker ressortorientiert handeln als parteipolitische Akteure (Nullmeier 2010: 258ff., vgl. Kapitel 4.6.3).

In Hessen wiederum agieren die Oberste Bauaufsicht im Wirtschaftsministerium und die Fachabteilung zur Wohnraumförderung im Umwelt- bzw. Wirtschaftsministerium in stärkerem Maße autonom. In der Novellierung der Bauordnung, aber auch in der Gestaltung neuer und der Überarbeitung bestehender Richtlinien zur Wohnraumförderung besteht vielfacher Austausch zum außerparlamentarischen Raum. Die ministeriellen Akteure nutzen dabei insbesondere in einem Low-Level Regelungsbereich mit geringer politischer Aufmerksamkeit ihre Möglichkeit zur strategischen Interaktion. Am stärksten gilt dies für die Instrumenten-Alternativen nach Typ 1, wo es um inkrementelle Veränderungen in bestehenden politischen Maßnahmen geht.

Formen der positiven Koordination, nach der formal zuständige Fachabteilungen andere inhaltlich betroffene Fachabteilungen frühzeitig einbeziehen, lassen sich für den barrierefreien bzw. -reduzierten Wohnraum nur sehr begrenzt nachweisen. In SachsenAnhalt und Hessen bestehen zwar mit Landesaktionsplänen zur Umsetzung der UNBRK interministerielle Strategiepapiere, die tatsächliche Praxis zum Disability Mainstreaming ist aber verhalten und es dominiert die klassische Ressortlogik. Insbesondere da, wo mutmaßliche Zielkonflikte auftreten - wie bei bezahlbarem und zugleich barrierefreiem Wohnraum - ist die interministerielle Zusammenarbeit beschränkt.

\section{Relevanz einzelner Individuen: Policy Entrepreneure?}

In beiden Bundesländern treten schließlich einzelne Individuen als aktive Veränderungsinteressen auf (IP 12: 58; IP 13: 3, 27; IP 20: 24; IP 54: 53). Hier ist kein kausaler Mechanismus zu einer Akteursgruppe erkennbar - vielmehr kommen solche Policy Entrepreneure entweder aus dem außerparlamentarischen oder aus dem parlamentarischen Raum. Akteure in Regierungsverantwortung verfügen dabei über eine stärkere Ressourcenausstattung zur Durchsetzung ihrer Interessen, wie das sachsen-anhaltische Beispiel des SPD-initiierten AUFZUGS-Programms beweist. Oppositionelle oder außerparlamentarische Policy Entrepreneure müssen diese fehlenden Ressourcen mit einem verstärkten Engagement, mit entsprechender Expertise oder guten Zugängen zum politisch-administrativen System kompensieren (IP 6: 69; IP 8: 45; IP 19: 3; IP 33: 14, 16). In Sachsen-Anhalt erweist sich eine Wohnberaterin durch ihr beständiges Einbringen in korporatistische Formate als eine solche Policy Entrepreneurin und in Hessen ist ein CBF-Mitglied zu nennen, welches als Sachverständiger zum barrierefreien Bauen über nachgewiesene Expertise verfügt. Auf ihn geht maßgeblich die nachträgliche Änderung des Bauordnungsentwurfs zur rollstuhlgerechten Nutzung von Aufzügen zurück (zur Rolle von Expertise vgl. auch Böcher/Töller 2007: 311).

Die Relevanz der personalen Ebene verstärkt die Eigendynamik und Zufälligkeit von politischen Prozessen. Hängen politische Vorhaben an einzelnen Personen, ist eine stete Beschäftigung im Falle eines Ruhestandes oder einer Erkrankung nicht gesichert (IP 
23: 92; IP 44: 12). Auch entscheiden hier noch stärker zwischenmenschliche Sympathien, wo sich Netzwerke zwischen den einzelnen Akteursgruppen entwickeln. Die hessischen Erfahrungen legen dabei nahe, dass diese nicht immer den formalen Zuständigkeiten entsprechen müssen: Weder die Behindertenbeauftragte, noch das Referat zur Umsetzung der UN-Behindertenrechtskonvention werden von einem Behindertenverband als tatsächliche Verbündete betrachtet (IP 46: 104). Entsprechend können Individuen nicht nur als Förderer*innen von neuen Policy-Ideen, sondern auch als Bremser*innen oder Verhinderer*innen fungieren. Zusammenfassend ist der AEP-Erklärungsfaktor »Akteure und ihre Handlungen « damit auch abhängig von empirisch schwer zu fassenden zwischenmenschlichen Faktoren, die einen maßgeblichen Einfluss auf den Verlauf politischer Prozesse haben können.

\section{Zentrale Erkenntnisse zu »Akteure und ihre Handlungen«}

Außerparlamentarische Interessengruppen fungieren als Veränderungsinteressen in ihrem Sinne. Während die Angebotsseite von Wohnraum eine weiche politische Steuerung durch Förderung und Information befürwortet, fordern insbesondere Behindertenverbände ein verbindlicheres Bauordnungsrecht. Die strukturelle Macht der Wohnungsund Bauwirtschaft wirkt dabei stärker und qualifiziert sie als »starke«Interessen.

Parteipolitische Akteure teilen offiziell das Ziel zu mehr barrierefreiem bzw. -reduziertem Wohnraum. Differenzen finden sich in der Priorisierung und in der Qualität des entsprechend herzustellenden Wohnraums, jedoch sind die Unterschiede zwischen den Fachpolitiken gewichtiger als zwischen den Parteien.

Die Ministerialverwaltung führt kritisch-loyal politische Zielvorgaben aus und verfügt dabei über ein großes Potential zur strategischen Interaktion. Dieses Potential kann durch enge politische Vorgaben oder personelle und budgetäre Restriktionen begrenzt werden.

Insgesamt handeln die Akteure auf Basis begrenzter Rationalität, was sich vor allem an der umstrittenen Wissenslage zu Mehrkosten der Barrierefreiheit zeigt. Sie verfolgen ihreZiele im Rahmen eines inkrementellen Politikstils und sehen sich, sofern sie ein Veränderungsinteresse verfolgen, starken Beharrungstendenzen des Status Quo ausgesetzt. 